\title{
Triaxial orbit-based model of NGC 4365
}

\author{
Remco C. E. van den Bosch, ${ }^{1}$ Glenn van de Ven, ${ }^{2}$ \\ Michele Cappellari ${ }^{1}$ and P. Tim de Zeeuw ${ }^{1}$ \\ ${ }^{1}$ Sterrewacht Leiden, Universiteit Leiden, Postbus 9513, 2300 RA Leiden, The Netherlands \\ ${ }^{2}$ Institute for Advanced Study, Einstein Drive, Princeton, NJ 08540, USA \\ email: Bosch@strw.leidenuniv.nl
}

\begin{abstract}
We have developed an orbit-based method for constructing triaxial models of elliptical galaxies, which fit their observed surface brightness and kinematics (van den Bosch et al.). We have tested this extended Schwarzschild method (1979) against analytical models with general distribution functions (DF) and find that we can recover the DF (van de Ven et al.). Here, we present a model of NGC 4365.
\end{abstract}

Keywords. galaxies: elliptical and lenticular, $\mathrm{cD}$ - galaxies: kinematics and dynamics

\section{NGC 4365}

NGC 4365 is an E3 galaxy with a kinematically de-coupled core (KDC). Its rotation axis is misaligned with respect to that of the main body of the galaxy (Surma \& Bender 1994; Davies et al. 2001; Statler et al. 2004). The bulk of the galaxy appears to rotate around the major axis, but the stars in the central $6^{\prime \prime}$ rotate around the minor axis. These properties can be explained by an intrinsically triaxial shape.

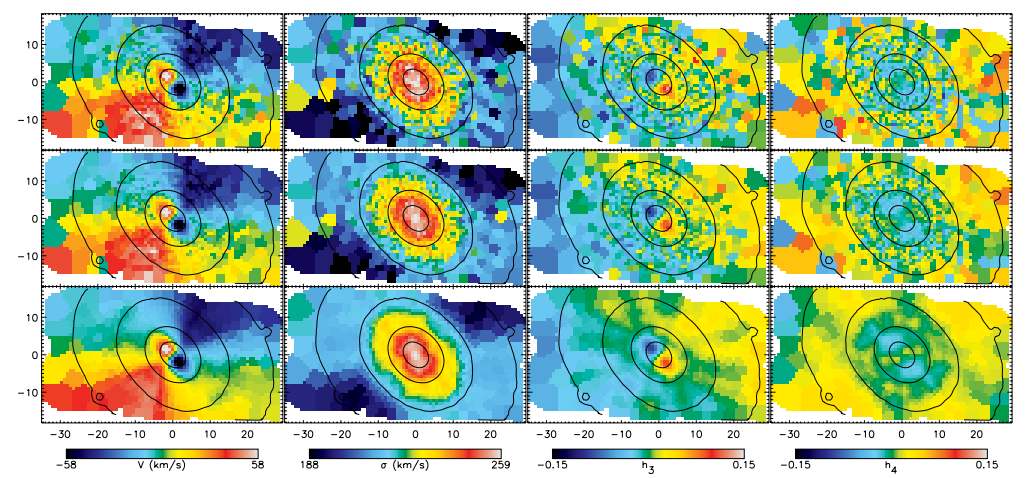

Figure 1. Stellar kinematics of NGC 4365 on the top row. From left to right: the mean velocity, velocity dispersion and the higher order moments $h_{3}$ and $h_{4}$ (measuring deviations from Gaussian). The contours are representative isophotes. The best-fitting model is shown on the bottom row and it is a good fit, reproducing the observed features in the symmetrized kinematics (middle row) with great accuracy $\left(\chi^{2} /\right.$ degrees-of-freedom $\left.\sim 1\right)$.

We measured the stellar kinematics of NGC 4365 (Fig. 1) with the SAURON integral-field spectrograph (Bacon et al. 2001). We constructed triaxial models using both the integralfield stellar kinematics and HST and ground-based imaging and including a central black hole. Our best-fit model (Fig. 1) is close to oblate with axis ratios of 1:0.97:0.73 (see also Statler et al. 2004) and surprisingly consists primarily of orbits that rotate around the short axis. 


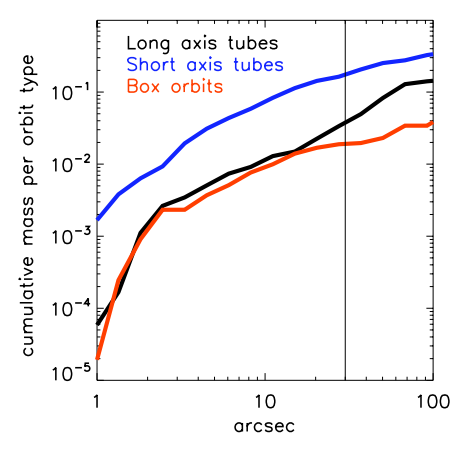

Figure 2. The cumulative mass per orbit-type as a function of radius. The modest fraction of stars on long axis tube orbits all have the same sense of rotation, and are responsible for the observed mean motion.

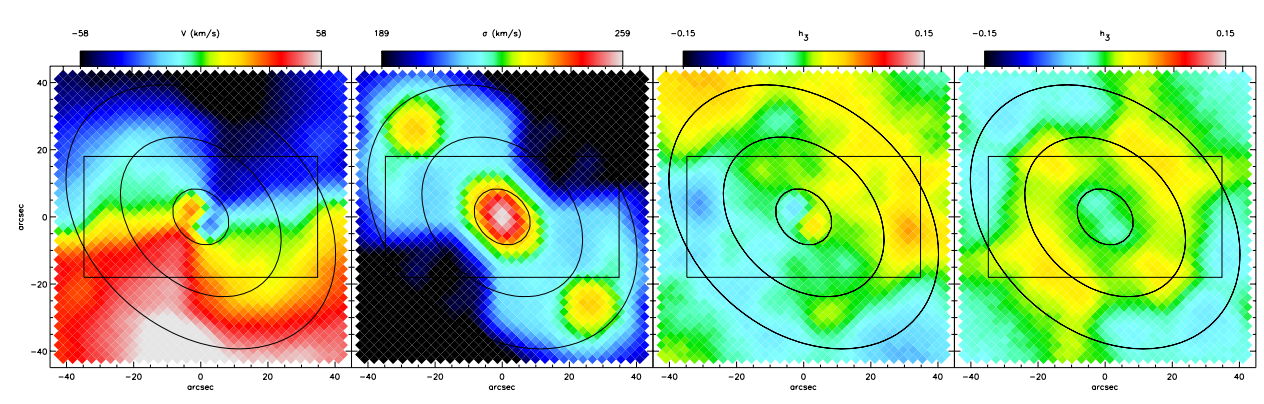

Figure 3. Kinematics of the model up to a radius of $60^{\prime \prime}$. Only the part inside the rectangular box is constrained by the kinematic observations, the rest is extrapolated. The kinematics outside the box are thus not very reliable, but they do allow one to see the extended structure.

\section{Orbit types}

In our best-fit model the short axis tube orbits account for $75 \%$ of the mass inside $30^{\prime \prime}$ (Fig. 2) and hence dominate even outside the KDC. This seems in contradiction with the rotation seen around the major axis at large radius in the observations. However, the average rotation of the stars populating the short axis orbits is almost negligible in this region, causing the high dispersion measured along the major axis. To be able to visualise the structure of the model we show the extended kinematics (Fig. 3). The extended structure, including the sigma dip along the minor axis and the continuation of the unaligned zero velocity curve show more clearly that the galaxy is oblate/triaxial.

\section{Acknowledgements}

RvdB thanks the Leids Kerkhoven-Bosscha Fonds for travel support.

\section{References}

Bacon R. et al. 2001, MNRAS, 326, 23

Davies R. L., et al. 2001, ApJ, 548, L33

Schwarzschild M. 1979, ApJ, 232, 2

Surma P. \& Bender R. 1995, A\&A, 298, 405

Statler T. S., Emsellem E., Peletier R. F. \& Bacon R. 2004, MNRAS, 353, 1

van den Bosch R. C. E., van de Ven G., Verolme E. K., Cappellari M. \& de Zeeuw P. T. 2006, MNRAS, submitted

van de Ven G., de Zeeuw P. T. \& van den Bosch R. C. E. 2006, MNRAS, submitted 